\title{
Hospital Rounds
}

National Cancer Institute

\section{Source}

National Cancer Institute. Hospital Rounds. NCI Thesaurus. Code C142165.

The act of seeing patients in the hospital, or another in-patient setting, to evaluate treatment, assess current course, and document the patient's progress. 